\title{
Pore-scale micro-computed-tomography imaging: Nonwetting-phase cluster-size distribution during drainage and imbibition
}

\author{
A. Georgiadis, ${ }^{1,2}$ S. Berg, ${ }^{1}$ A. Makurat,${ }^{1}$ G. Maitland ${ }^{2}$ and H. Ott ${ }^{1,3, *}$ \\ ${ }^{1}$ Shell Global Solutions International BV, Rijswijk, The Netherlands \\ ${ }^{2}$ Department of Chemical Engineering, Imperial College London, United Kingdom \\ ${ }^{3}$ Department of Earth Science \& Engineering, Imperial College London, United Kingdom
}

(Received 21 March 2013; published 4 September 2013)

\begin{abstract}
We investigated the cluster-size distribution of the residual nonwetting phase in a sintered glass-bead porous medium at two-phase flow conditions, by means of micro-computed-tomography $(\mu \mathrm{CT})$ imaging with pore-scale resolution. Cluster-size distribution functions and cluster volumes were obtained by image analysis for a range of injected pore volumes under both imbibition and drainage conditions; the field of view was larger than the porosity-based representative elementary volume (REV). We did not attempt to make a definition for a two-phase REV but used the nonwetting-phase cluster-size distribution as an indicator. Most of the nonwettingphase total volume was found to be contained in clusters that were one to two orders of magnitude larger than the porosity-based REV. The largest observed clusters in fact ranged in volume from $65 \%$ to $99 \%$ of the entire nonwetting phase in the field of view. As a consequence, the largest clusters observed were statistically not represented and were found to be smaller than the estimated maximum cluster length. The results indicate that the two-phase REV is larger than the field of view attainable by $\mu \mathrm{CT}$ scanning, at a resolution which allows for the accurate determination of cluster connectivity.
\end{abstract}

DOI: 10.1103/PhysRevE.88.033002

PACS number(s): 47.56.+r, 47.55.nb, 81.70.Tx

\section{INTRODUCTION}

Multiphase flow in porous media is highly relevant to geology, hydro-geology, and petroleum engineering disciplines. This is usually described on a macroscopic level with continuum mechanics approaches, often based on empirical observations such as the two-phase extension of Darcy's law [1-5], which lacks a fundamental basis [6]. Most approaches in porous-media flow depend on indirect and/or macroscopic observations. These macroscopic flow properties, however, are ultimately defined by microscopic processes, i.e., fluid flow at the pore scale (see, e.g., Ref. [7]). There is therefore significant interest in the direct observation of the pore-scale fluid behavior, aimed at determining how macroscopic properties depend on the pore-scale distribution of the associated fluid phases and defining a representative elementary volume (REV) for two-phase flow, important for numerical simulations and experimental imaging techniques.

Over the past decade, micro-computed-tomography $(\mu \mathrm{CT}$; synchrotron-based and bench-top instruments) has become a frequently used technique to study porous media and geological samples [8-11]. Together with nuclear resonance (MRI) and acoustic techniques, X-ray-based tomography is one of the few nondestructive techniques that give detailed insight into the structure of porous media over length scales ranging down to a few hundred nanometers $[12,13]$. The resolution of MRI depends primarily on the strength of the applied magnetic field

*holger.ott@shell.com; h.ott@imperial.ac.uk

Published by the American Physical Society under the terms of the Creative Commons Attribution 3.0 License. Further distribution of this work must maintain attribution to the author(s) and the published article's title, journal citation, and DOI. gradient and can theoretically be increased to the wavelength of the scattering vector. Currently MRI voxel resolution is limited to around $0.05 \times 0.05 \times 1 \mathrm{~mm}$, which is not sufficient to image the pore scale [14]. At the pore scale, $\mu \mathrm{CT}$ images of real rocks have been used to construct three-dimensional (3D) networks for modeling of fluid flow by pore-network modeling [15], but also for direct modeling of fluid flow in the pore space, by, e.g., Lattice Boltzmann [16] or Stokes flow [17] methods.

A fundamental understanding of fluid flow in porous media, and the verification of pore-scale modeling, however, requires direct observation of different fluid phases in the pore space. Currently, the state of the art method is imaging of static fluid distributions at pore scale, which is not entirely new. The study by Coles et al. [18,19] in 1998 combined synchrotron-based pore-scale imaging of oil and water, coupled to numerical simulations with a Lattice Boltzmann code. Since 1998 a rapid development in $\mu \mathrm{CT}$ has made the quality and spatial resolution of the 1998 synchrotron capabilities currently available for laboratory use with bench-top $\mu \mathrm{CT}$ scanners.

Youssef et al. [20,21] conducted a $\mu \mathrm{CT}$ study on Fontainebleau sandstone at spatial resolutions of 10 and $5 \mu \mathrm{m}$ while conducting a two-phase flow experiment. The trapped nonwetting phase distribution was imaged as a function of interfacial tension. The observation represents capillary desaturation, i.e., the decrease of the residual nonwetting phase saturation with increasing capillary number $\left(\mathrm{Ca}>10^{-5}\right)$, which is the principle often employed in enhanced oil recovery processes. Somewhat similar studies were conducted by the ANU group [22,23], who investigated the spatial distribution of a residual gas phase in Fontainebleau sandstone, and by Iglauer et al. [24] who studied Clashach and Doddington sandstones, following an oil-brine drainage-imbibition sequence. The observations were similar and qualitatively confirm earlier observations in two-dimensional micromodels [25] showing 
that over substantial saturation ranges, the nonwetting phase does not flow as a continuous phase, but as distinct ganglia. The size distribution functions show that these ganglia are not restricted to the pore scale but can extend over many pores ranging up to the length scale of the sample. This is an interesting finding, in particular relating to the fundamental concept of the representative elementary volume (REV) as introduced by Bear in 1972 [4] (see also Ref. [26]). In porous-media flow, the transition from pore-scale physics to continuum mechanics is performed by averaging over an REV. This concept is based on the observation that in homogeneous rocks, at a certain length scale and above, average properties such as the porosity of a sample become independent of the averaging volume. While this concept may apply in single-phase flow, it is not clear if it holds for multiphase flow, as clusters may span over many length scales.

Interfacial properties of fluids in porous-media flow largely determine macroscopic properties; their effect is commonly not taken into account explicitly, but lumped together with other pore-scale properties. Recent work on linking between pore- and core-scale observations [27-30] indicates that advances in imaging techniques and analysis allows for explicit accounting of such local pore-scale effects.

In the present study we investigated the development of $n$-decane clusters in a glass-bead porous medium by means of $\mu \mathrm{CT}$ imaging with pore-scale resolution. We obtained clustersize distribution functions and cluster volumes by image analysis, for a range of injected pore volumes, under both imbibition and drainage conditions; the field of view was larger than the porosity-based REV. Porous glass was used as a model rock with well-defined surface properties and suitable pore size for $\mu \mathrm{CT}$ imaging. $n$-decane was used as a model for the nonwetting phase, and the $n$-decane-brine interfacial tension $\gamma$ was varied by surfactant addition over a range of values representative for $\mathrm{CO}_{2}$-brine systems [31-35]. The focus of the study was the detailed cluster analysis and the comparison of single-phase REV with the characteristic cluster-size and saturation distribution in both drainage and imbibition.

\section{MATERIALS AND METHODS}

\section{A. Porous medium and $\mu \mathrm{CT}$ imaging}

The flow experiments were carried out using a sintered glass-bead sample (core) with $10 \mathrm{~mm}$ diameter and a length of $20 \mathrm{~mm}$. The porosity and permeability of the sample was experimentally determined at $\phi=0.318$ and $K=20-24 \mathrm{D}$, respectively. We embedded the core in a polycarbonate core holder by a shrinking process for a tight fit to avoid fluid bypassing. A vertical flow geometry was used with fluid injection from top to bottom. The fluids were injected by means of two high-precision displacement pumps (Quizix), one dedicated for the $n$-decane and the other for the brine phase. The connections from the injection pumps to the core holder and the production vessel were made of flexible PTFE tubing allowing a full $360^{\circ}$ rotation of the core as necessary for the tomographic scans. All wetted parts were thoroughly cleaned by sonication in isopropanol (purity $>99.5 \%$ ) to guarantee well-defined interfacial properties during the experiments.
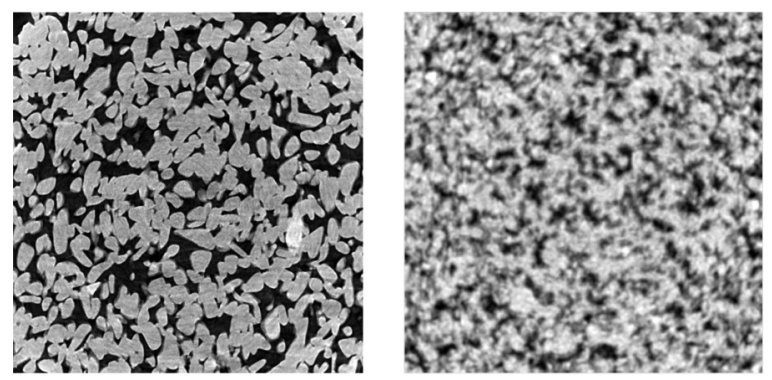

FIG. 1. Cross section of a high-resolution $\mu \mathrm{CT}$ scan of the porous glass sample at $4.2 \mu \mathrm{m} /$ voxel and $3 \mathrm{~h}$ scanning time with an edge length of $1.6 \mathrm{~mm}$ (left), and of a scan with a resolution as actually used throughout this study with $11.5 \mu \mathrm{m} /$ voxel and 10-20 min scanning time (right). The edge length is $5.1 \mathrm{~mm}$.

The core holder was placed in a $\mu \mathrm{CT}$ scanner (CT-ALPHA, ProCon X-Ray $\mathrm{GmbH}$ ) for in situ imaging of the pore space and the fluid distribution in the pores during the core flood. The scanner was operated at $100 \mathrm{kV}$ and $60 \mu \mathrm{A}$, which gave a good contrast between the fluids and porous medium of the given relatively large sample size. We recorded $\mu \mathrm{CT}$ scans at a resolution of $4.2 \mu \mathrm{m}$ (voxel size) for characterization of the pore space and at $11.5 \mu \mathrm{m}$ during two-phase flow experiments. Figure 1 shows a high-resolution $\mu \mathrm{CT}$ cross section of the porous glass sample on the left. At $4.2 \mu \mathrm{m}$ resolution the grain and pore space are resolved in great detail. This detail is lost by increasing the voxel size to $11.5 \mu \mathrm{m}$, but a larger field of view is gained, which is essential for two-phase flow experiments as will be discussed further in the following section. The compromise between high spatial resolution and large field of view is adjusted as long as the ability to determine whether the pore space or fluid clusters are connected or not is maintained, information which is used in the present study.

A 3D subvolume of the porous glass at high resolution (left side) and the extracted pore space (right side) are shown in Fig. 2; individual pores are colored differently. In further characterizing the porous medium, and subsequently the fluid distribution in the pore space, several steps of data analysis were involved. In general the following steps were required: reconstruction of the tomographic image, image filtering, segmentation of pore space and fluid phases, skeletonization, and network extraction or statistical cluster analysis. Depending on the experiment and application, not all steps are required. For the high-resolution scan of the present study the pore space was segmented by a water shading algorithm (Avizo).
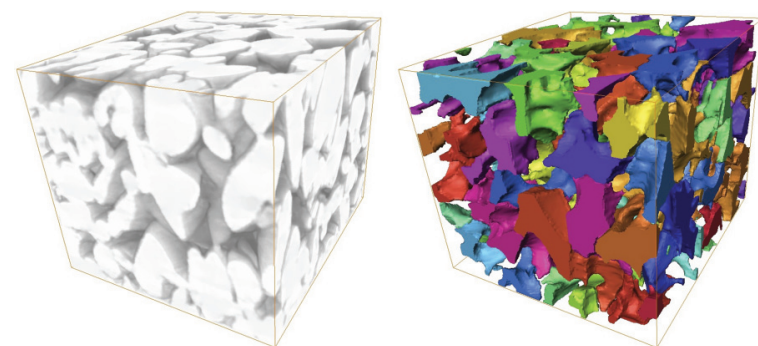

FIG. 2. (Color online) High-resolution image of the porous glass sample (left) and the respective pore space (right) rendered with Avizo. 




FIG. 3. (Color online) Pore radius distribution calculated from the high- and flooding-resolution scan settings from the same sample shown in Fig. 1.

For the flooding-resolution scans the raw images acquired were filtered by an FFT bandpass filter and segmented by thresholding using Li's method (ImageJ) as further outlined below.

In Fig. 3 the pore-radius distribution (computed with the Avizo XSkeleton package, which is a skeletonization package that uses an Euclidean distance map and thinning) as extracted from the flooding- and the high-resolution scans of the dry sample are displayed. The distributions are quite similar, with the flooding-resolution distribution being slightly broader, validating the workflow (scan settings and image analysis) for the flooding-resolution scans.

Based on the segmented pore space, we estimated the permeability using a single-phase flow numerical simulation, and compared the results to the experimentally determined permeability. We computed the apparent permeability (Avizo plug-in) by simulating a flow experiment in a specific spatial direction by attaching inlet and outlet distribution elements. The simulation used a finite volume scheme to simulate Stokes flow and was performed on the segmented pore structure on a length scale larger than the porosity-based REV (discussed below). A snapshot of the simulation is displayed in Fig. 4 where the flow was from right to left, by applying a pressure

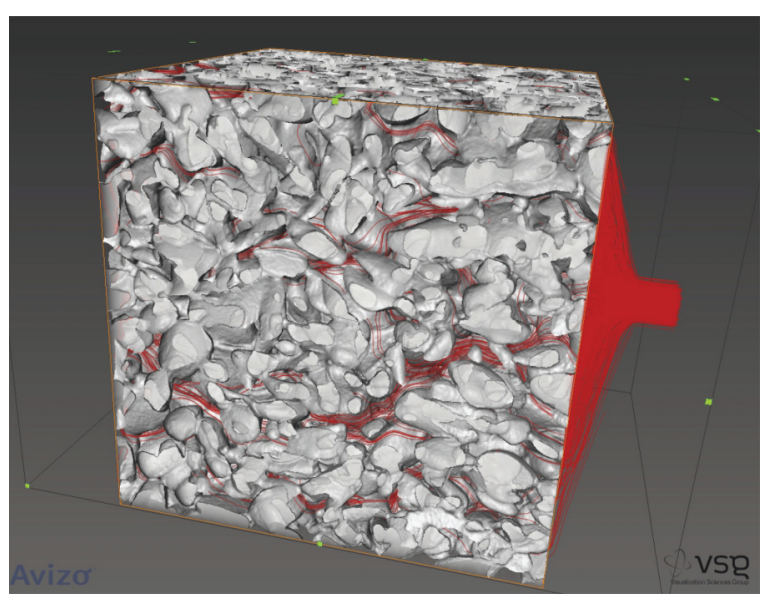

FIG. 4. (Color online) Snapshot of a single-phase stokes flow simulation in a segmented volume larger than the single-phase REV. Red lines represent the flow lines. The injection is from right to left. gradient of $0.03 \mathrm{MPa}$, and using a fluid of $1 \mathrm{cP}$ viscosity. The apparent permeability was computed at $15.5 \mathrm{D}$, which is in the same permeability range as, and hence in agreement with, the experimental results of $K=20-24 \mathrm{D}$, measured on larger samples. Potential reasons for the difference between simulated and experimental values could be sample heterogeneity; medical CT scans of the sintered glass-bead showed porosity variations in the order of $10 \%-20 \%$ on a larger length scale, which can lead to a large permeability variation at the smaller length scale. This would be in addition to uncertainties in the image analysis, e.g., filtering and thresholding.

\section{B. Two-phase flow experiments}

During the core flood experiments, nonwetting and wetting fluid phases were injected in different sequences. $n$-decane (purity $>99 \%$ ) was used as the nonwetting phase. The aqueous phase (wetting phase) used was demineralized water with 4 wt $\% \mathrm{CsCl}$ (purity $>99 \%$ ) doping for enhancing the fluid-fluid $\mu \mathrm{CT}$ contrast. The surfactant docecyl trimethyl ammonium bromide (DTAB, purity $>99 \%$ ) was introduced into the aqueous phase for selected experiments at concentrations ranging both above and below the critical micelle concentration. The addition of DTAB allowed a lowering of the interfacial tension, representing changes equivalent to those that would occur in the $\mathrm{CO}_{2}$-brine system over different thermodynamic conditions [31-35], but without influencing other fluid properties such as density and viscosity. The $n$-decane-DTAB-brine interfacial tension was measured using the du Noüy ring method.

Prior to every experiment, the glass-bead core was cleaned by flooding at least $200 \mathrm{PV}$ (pore volumes) of isopropanol and was subsequently dried under vacuum. Before fluid injection, the core was scanned in a dry state. Subsequently, the sample was saturated with brine and/or $n$-decane under initial vacuum condition and finally flooded at $0.5 \mathrm{MPa}$ pore pressure. All ensuing flooding experiments were performed at a constant flow rate of $1 \mathrm{ml} / \mathrm{min}$, with a back pressure of $0.2 \mathrm{MPa}$. During the $\mu \mathrm{CT}$ scans injection was ceased and the inlet and outlet valves were closed. $\mu \mathrm{CT}$ scans at three different positions along the core were then recorded, each requiring approximately $20 \mathrm{~min}$ to complete. Following the completion of the scans for all three positions, injection continued until a different PV value was obtained and $\mu \mathrm{CT}$ scans were repeated.

\section{EXPERIMENTAL RESULTS}

\section{A. Image processing}

During the core flood experiments, $\mu \mathrm{CT}$ scans were recorded at flooding resolution. The raw images acquired were filtered to reduce noise and then segmented to extract the pore space and fluid saturation. The data processing steps are shown in Fig. 5. The upper row shows a $\mu \mathrm{CT}$ cross section of the sample under vacuum, and from left to right: raw image, FFT filtered image with the upper and lower threshold being set at 5 and 640 voxels, respectively, and image segmented by Li thresholding using ImageJ. The 3D pore space was derived from the segmented vacuum data sets, giving a porosity of 0.327 , which is in good agreement with the experimentally measured porosity of 0.318 . This, together with 
raw image

vacuum


FFT filter

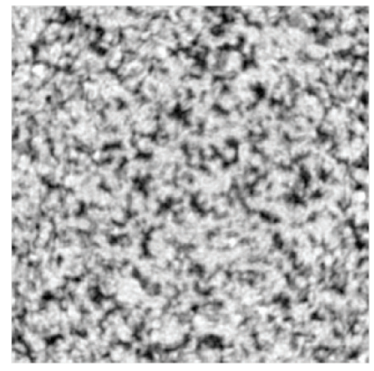

segmentation
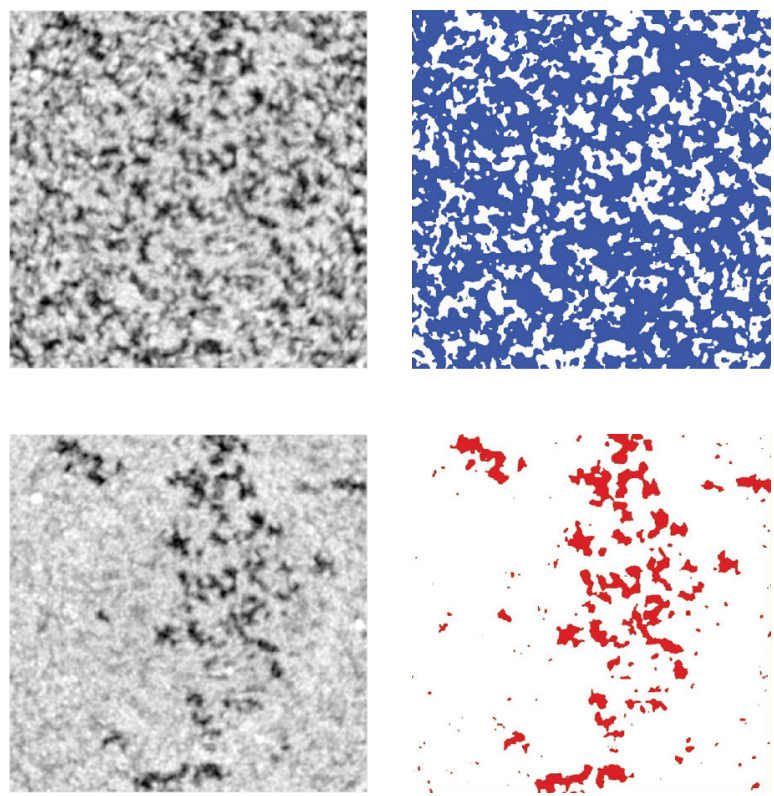

combined

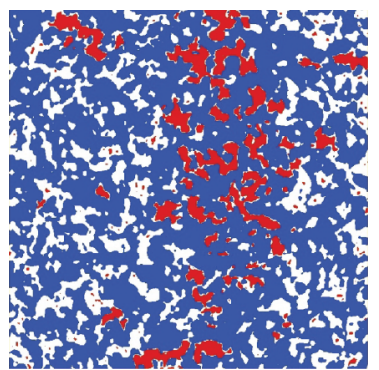

3D representation

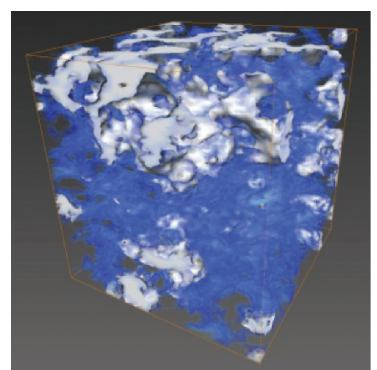

FIG. 5. (Color online) Upper row: $\mu \mathrm{CT}$ cross section in the dry state (flooding-resolution, under vacuum). Lower row: $\mu \mathrm{CT}$ cross section of the sample saturated with $n$-decane-brine (same slice). From left to right: raw images, images after FFT filtering and images after segmentation. In the upper right, segmented images of the pore space and the fluid phase are superimposed. The lower right image shows the 3D visualization of the fluid phases: brine in blue and $n$-decane in white.

the well-reproduced permeability and pore-radius distribution (as discussed in Sec. II A), validated the image filtering and thresholding applied. The lower row of Fig. 5 shows the same cross section after injection of $1 \mathrm{PV}$ of $n$-decane in the initially brine-saturated sample (primary drainage). To achieve a good $\mathrm{X}$-ray contrast, the brine phase was doped with $\mathrm{CsCl}$ at a concentration where the contrast between glass beads and brine is minimized, achieving a clear contrast between the glass-brine initial system and the injected $n$-decane system. As a consequence, the nonwetting phase volume can be directly derived by segmentation with a single threshold. The nonwetting phase clusters in this work were all derived from the segmentation of the $\mu \mathrm{CT}$ data taken at the respective saturation states. The cross section of the pore space combined with the extracted nonwetting phase distribution and a 3D representation of both fluid phases (with the porous medium being transparent) are shown in the rightmost images in Fig. 5.

\section{B. Cluster analysis}

A range of flooding experiments were conducted by varying (a) the flooding sequence to establish the initial saturation and (b) the concentration of DTAB to adjust the interfacial tension corresponding to capillary number $\left(\mathrm{Ca}\right.$ ) ranging from $10^{-5}$ to $10^{-4}$. The nonwetting phase saturations obtained for different capillary numbers are within a range in which the residual saturation starts to become a function of $\mathrm{Ca}$ (see capillary desaturation curve [5]).

For the experiments I $(\gamma=50.5 \mathrm{mN} / \mathrm{m})$, IV $(\gamma=$ $8.6 \mathrm{mN} / \mathrm{m})$, and VI $(\gamma=27.2 \mathrm{mN} / \mathrm{m})$ the core was presaturated with brine and subsequently drained with $200 \mathrm{PV}$ $n$-decane (primary drainage) before the imbibition was started.
In experiments II $(\gamma=50.5 \mathrm{mN} / \mathrm{m}), \operatorname{III}(\gamma=8.6 \mathrm{mN} / \mathrm{m})$, and $\mathrm{V}(\gamma=27.2 \mathrm{mN} / \mathrm{m})$ the sample was initially presaturated with $n$-decane and brine was injected thereafter. $\mu \mathrm{CT}$ scans were recorded from the respective initial state and after injection of volumes as indicated in the respective figures (in PV).

In Fig. 6 the 3D fluid distribution derived from the segmented images during drainage is shown. The brine is displayed in blue and the $n$-decane in white, showing the growth of the $n$-decane clusters as a function of injected volume. The flow direction is from top to bottom. After the injection of $1 \mathrm{PV}$, clusters of different sizes spanning across multiple pores are clearly visible. The image on the right shows the same distribution, but with the individual clusters colored differently, visualizing clusters of different sizes (rotated view). The larger clusters show a disklike shape with the truncated length along the flow direction. The continued injection leads to a clear increase of $n$-decane saturation.

Of particular interest is the size and the volume of the various trapped clusters. Their volume and length distributions are shown in Fig. 7 on a double logarithmic scale. The cluster-volume distribution shows more scatter than the length distribution, following a power-law behavior in accordance with invasion percolation theory $[36,37]$ given by the form

$$
N(s) \propto s^{-\tau},
$$

where $N(s)$ is the number frequency of cluster size $s$. An exponent $\tau=2.189$ has been found by Monte Carlo simulation on different lattices [38], which best agrees with most of the cluster-size distribution obtained. Deviation from the power-law behavior was observed for large clusters, a fact associated with clusters growing at values away from the percolation threshold [38]. A power law with exponent 

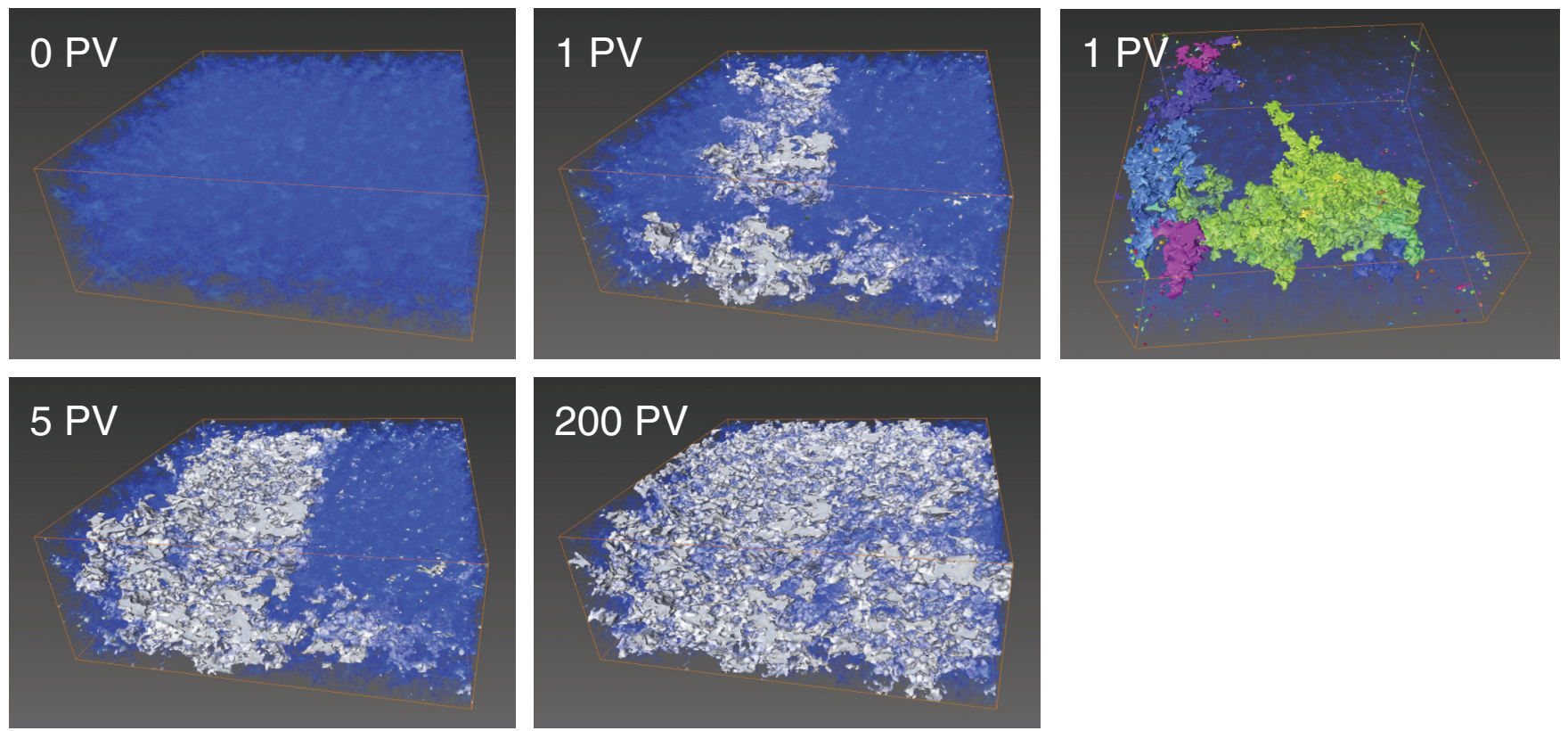

FIG. 6. (Color online) 3D fluid saturations during drainage. The images are recorded with $11.5 \mu \mathrm{m} /$ voxel. The length of the base line corresponds to $7.6 \mathrm{~mm}$. Clearly visible is the growth of the $n$-decane clusters (white). The image on the top right shows the $n$-decane clusters individually colored after injection of $1 \mathrm{PV} n$-decane (the image is rotated with respect to the image on the left).

$\tau=2.189$ is also plotted in Fig. 7 showing agreement with the results obtained in the present study, but also the range where the larger cluster distribution deviates. Comparable observations were made by Iglauer et al., who obtained an exponent $\tau=2.05$ for ( $n$-octane + brine) system in water-wet sandstone [24], and $\tau=2.12$ for ( $n$-decane + brine) system in oil-wet sandstone [39]. Pentland et al. [40] found exponents ranging from (2.004 to 2.0137) for $\mathrm{CO}_{2}$ trapped phases in brine-saturated glass and sand packs, at reservoir conditions. It should be noted that the total volume of the largest clusters may be underestimated due to possible truncation associated with field of view limitations.

The cluster-length distribution, shown in the lower part of Fig. 7, corresponds to the diagonal length of the cluster bounding box. The data appear less scattered than the volume distribution, and both show characteristics of a power-law behavior. In order to put the cluster lengths into perspective, the pore radius distribution and the single-phase porosity-based REV are also displayed. Even the smaller clusters are clearly dispersed over several pore lengths, and apparently there are clusters extending even far beyond the porosity-based REV, which raises the question about the two-phase flow; an inherent attribute of the $\mu \mathrm{CT}$ analysis arises from the fact that the REV for two-phase flow is larger than the field of view (see discussion in Secs. III C and III D).

Figure 7 clearly shows that the cluster size does show a power-law distribution only for the smaller clusters but deviates from the power-law distribution at larger cluster sizes. Since data for a larger field of view are inaccessible without losing resolution (required to determine the connectivity of clusters), we can only speculate whether for a larger field of view the power-law range would increase or even span the whole dynamic range and thus indicate a two-phase REV. Instead we rather concentrate on some implications on the non-power-law tail of the cluster size distribution and the computation of the power-law exponent. Since $N(s)$ displayed in Fig. 7 spans more than three orders of magnitude, in a power-law fit the much larger $N(s)$ for the smaller $s$ would have a much higher weight. Therefore, commonly the $M(s)$ distribution, given by the form

$$
M(s)=\sum_{s=s^{\prime}}^{\infty} s^{\prime} N\left(s^{\prime}\right) \propto s^{2-\tau},
$$

is used [24] because $M(s)$ varies by less than one order of magnitude. Computing the $M(s)$ according to Eq. (2), however, implicitly assumes that $N(s)$ has the form of a power-law distribution over the whole range, which is not the case. When computing the $M(s)$ the sum also includes the non-power-law tail with a non-negligible weight. That is illustrated for one data set taken as example (1 PV, drainage) displayed in Fig. 8. In the top panel we show the $N(s)$ distribution, and a power law superimposed at an exponent of $\tau=1.95$ that visually fits the data over the largest range. The $M(s)$ distribution computed over the whole range displayed in the bottom panel is well fitted with an exponent of $\tau=2.23$, which is still compatible with the $N(s)$ data but not an optimum fit anymore. Truncating the $N(s)$ distribution when computing the $M(s)$, cutting off the non-power-law tail (vertical line in top panel), leads then to a very different $M(s)$ fitted with $\tau=2.34$, which clearly shows that the non-power-law tail has a large influence on the $M(s)$ and the fitting exponent. It therefore becomes questionable whether fitting the $M(s)$ for a distribution with non-power-law tails leads to reliable results.

Apart from that, already the binning when computing the $N(s)$ histogram has an influence on the power-law exponent. The maximum likelihood estimator $[41,42]$ is a direct method that does not rely on binning or the computation of $N(s)$ 

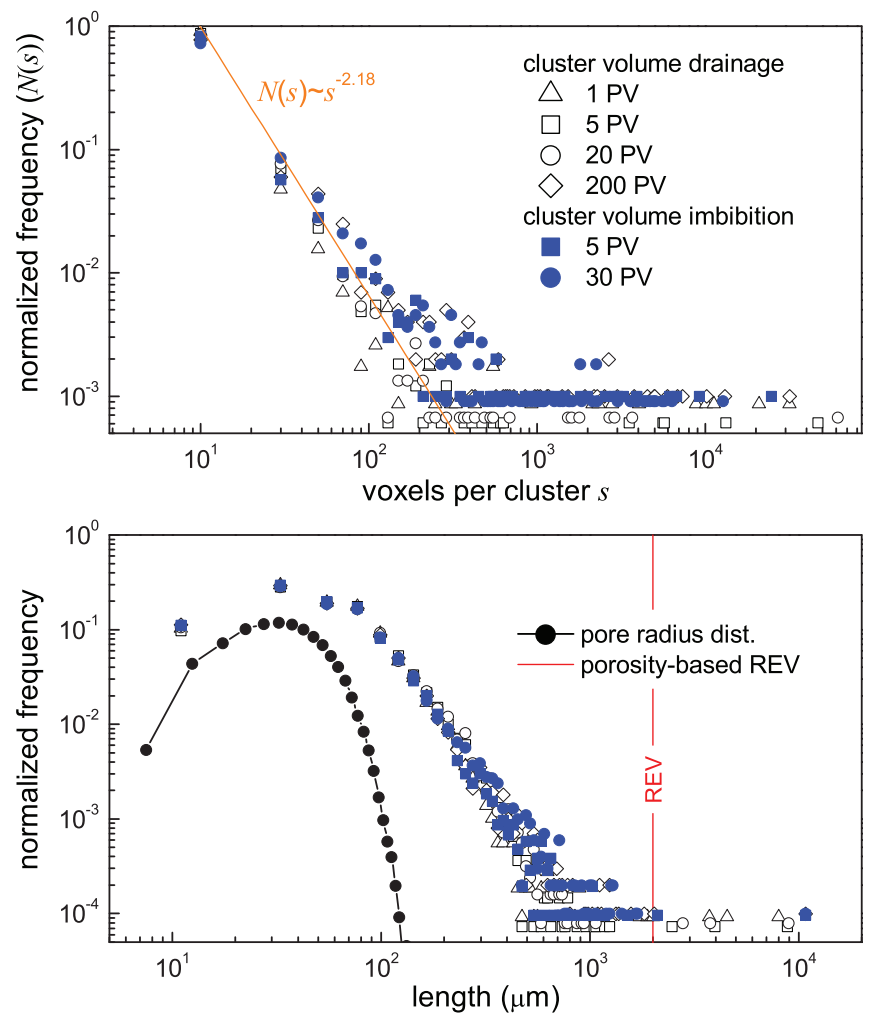

FIG. 7. (Color online) Top: normalized volume distribution of the nonwetting phase ( $n$-decane) clusters. Bottom: pore-radius distribution compared to the cluster-length distribution. Data of different pore volumes of injected $n$-decane during drainage and imbibition are plotted.

and $M(s)$. The associated power law computed using the MATLAB ${ }^{\circledR}$ script from Refs. [41,42] for the cumulative distribution $M(s)$ displayed in Fig. 8 is shown with a red continuous line. The power-law exponent estimated in this way is $\tau=2.10$, which shows very good agreement with the data. We performed this data analysis for all data sets in our study and display the resulting exponents in Fig. 9. The exponents obtained had a standard deviation of 0.11 , which is in the same range as the exponents between 1.95 and 2.2. All are somewhat compatible with the $N(s)$ data in Fig. 8 and present an estimate of the uncertainty in the data. Within this uncertainty, no clear trends are visible, and overall the data are, within the uncertainty, compatible with a percolation model that predicts exponents of $\tau=2.189$.

In Fig. 10 the volume of the largest cluster is plotted versus the total nonwetting phase saturation. For both drainage and imbibition, the data follow a similar trend. The volume of the largest cluster grows monotonically with the nonwetting phase saturation and shows relative values between 65 to $99 \%$; i.e., the majority of the nonwetting phase is contained in the largest cluster, in agreement with similar findings in the literature $[43,44]$. This is significant as the largest clusters in a porous medium bear the highest potential of being mobilised. Induced pressure gradients created by viscous forces during flow will act along the length of a large cluster more effectively compared to smaller ones, thus potentially rendering more mobile the majority of the nonwetting trapped phase. The lower graph of Fig. 10 shows the behavior of the
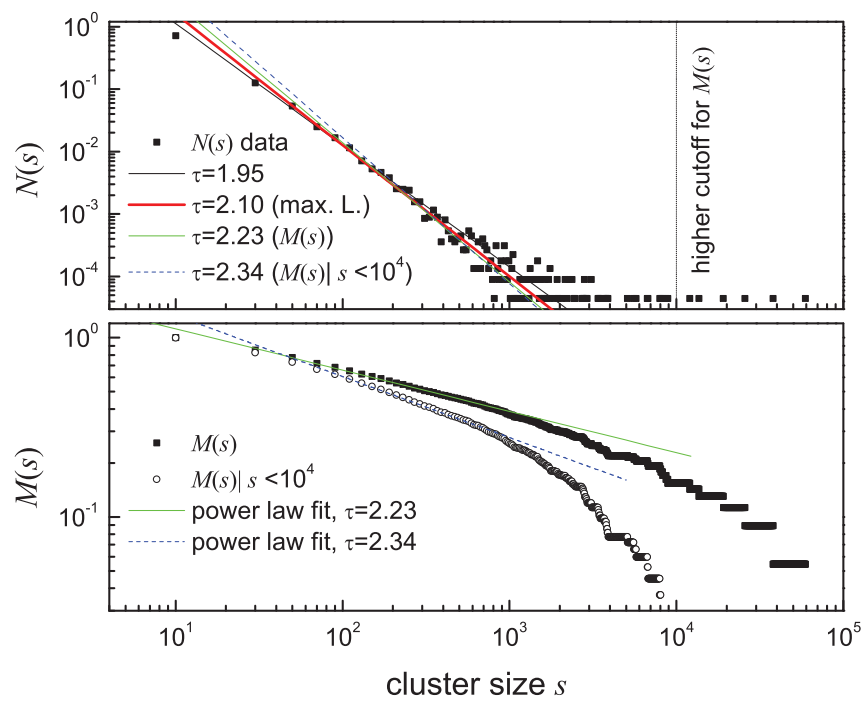

FIG. 8. (Color online) Normalized cluster volume (top) and cumulative distribution (bottom) for different regions of interest. Computed exponent $\tau=2.10$ (thick red line) derived by maximum likelihood (max. L.) estimator for power-law determination using the MATLAB $^{\circledR}$ script from Refs. [41,42].

six largest clusters in drainage and imbibition as a function of the nonwetting phase saturation. When the nonwetting phase saturation is increased (drainage), the largest cluster grows at the expense of smaller clusters. A similar behavior, but in the reverse direction, is observed during imbibition. When the nonwetting phase saturation is decreased (imbibition), the largest cluster breaks apart and fragments into smaller clusters.

\section{Cluster length estimation from $\mathrm{Ca}$}

In the course of this study we observed clusters with lengths over the range of several millimeters, i.e., range of the dimension of the experiment $(10 \mathrm{~mm})$. An interesting question is whether or not the observed cutoff is a property of the finite size of the experiment (sample and field of view), as we would expect for a true power-law behavior suggesting clusters on all length scales, or whether there is a maximum cluster size that cannot be exceeded independent of the size of the experiment. Both cases lead to a dilemma, since a further

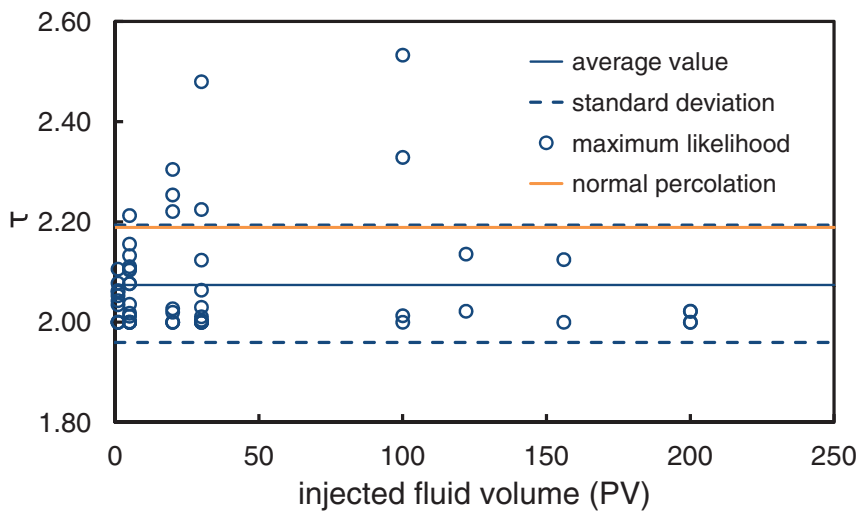

FIG. 9. (Color online) Power-law exponents fitted to the individual data sets using the maximum likelihood estimator [41,42], as a function of injected pore volumes. 

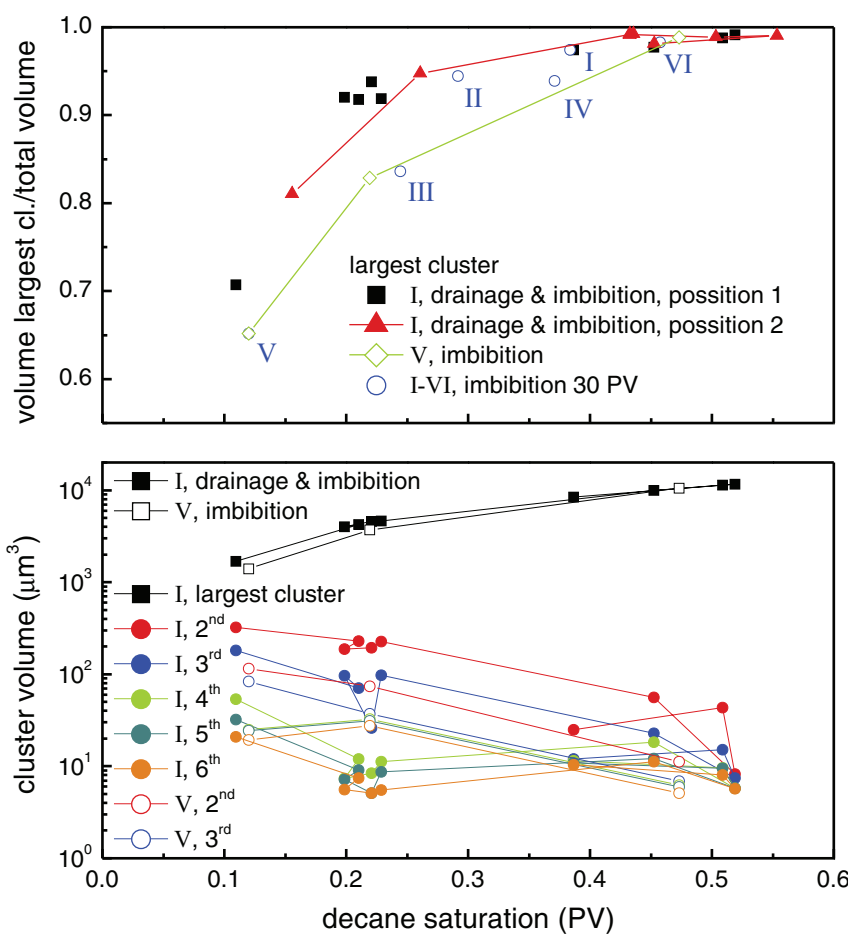

FIG. 10. (Color online) Cluster volume development during drainage and imbibition. Top: volume of the largest $n$-decane cluster over the total $n$-decane volume in the field of view. Bottom: length of the six largest clusters as a function of brine saturation for different drainage and imbibition cycles.

increase of sample and field of view would lead to a decrease of CT resolution, and consequently to the inability to decide whether an observed cluster is actually connected or not.

According to Hilfer and Øren [45], a maximum cluster length can be estimated via the macroscopic capillary number $\overline{C a}$, defined as the ratio between the viscous $F_{\mathrm{visc}}=A_{\mathrm{cl}} \Delta P=$ $A_{\mathrm{cl}} l_{\mathrm{cl}} \mu_{\mathrm{w}} \nu_{\text {Darcy }} / K$ and capillary $F_{\text {cap }}=A_{\mathrm{cl}} P_{\mathrm{b}}$ forces, given by the form

$$
\overline{C a}=\frac{l_{\mathrm{cl}} \mu_{\mathrm{w}} \nu_{\text {Darcy }}}{K P_{\mathrm{b}}},
$$

where $A_{\mathrm{cl}}$ is the cluster cross-sectional area, $l_{\mathrm{cl}}$ the cluster length along the viscous pressure drop, $K$ the permeability, $\mu_{\mathrm{w}}$ the viscosity of the aqueous phase, $v_{\text {Darcy }}$ the Darcy velocity, and $P_{b}$ the breakthrough pressure from the capillary pressure curve, defined as $P_{\mathrm{b}}=P_{\mathrm{c}}\left(S_{\mathrm{w}}=S_{\mathrm{b}}\right)$. Unlike the microscopic capillary number, $\overline{C a}$ depends explicitly on the length scale, i.e., on the cluster size. For a mobile cluster the viscous forces would overcome capillary forces, thus $\overline{C a}>1$. By rearranging the terms in this inequality the maximum cluster length $l_{\mathrm{cl}, \max }$ may be determined by the form

$$
l_{\mathrm{cl}, \max }<\frac{P_{\mathrm{b}} K}{\mu_{\mathrm{w}} v_{\text {Darcy }}} .
$$

The maximum cluster size is estimated to be in the order of $0.2 \mathrm{~m}$ and thus one order of magnitude larger than the total size of the experiment for this specific porous medium, i.e., the larger clusters are likely to be truncated by the size of the experiment.



FIG. 11. (Color online) Porosity and $n$-decane saturation as a function of averaging window.

\section{Representative elementary volume}

As illustrated in Figs. 7 and 10, there are only few large nonwetting phase clusters in the field of view with a size in the order of the experimental dimensions (or even larger). This fact raises immediately the question of how representative this particular field of view is for the representation of a two-phase system. In Fig. 11 the porosity and the total nonwetting phase saturation are shown as a function of the averaging window. The porosity data indicate an REV around 2-3 $\mathrm{mm}$ (the high resolution scan gives about $2 \mathrm{~mm}$ ), but at this length scale the saturation still increases substantially, indicating that a two-phase REV is substantially larger, also consistent with the observations in Secs. III C and III E.

This is best illustrated in Fig. 6, where the nonwetting phase saturation is obviously determined by only a few large clusters in the field of view. Since we have established from the porosity that our field of view covered more than twice the length of the REV, we should be able to take 8 subsamples and find the same saturation value. But as is obvious from Fig. 6, this depends entirely on the sampling. For $5 \mathrm{PV}$ injected, for instance, when a sample cube with half length from the upper left corner at the front is selected, a high nonwetting phase saturation is obtained. However, if the same volume is sampled from the upper right corner at the back, a very low nonwetting phase saturation is found, which is reflected in the increase of the saturation in Fig. 11 beyond the porosity-based REV. While the porosity becomes almost constant for averaging windows that are approximately half the field of view, the saturation still shows a large variation. This means in practice that a two-phase REV can be much larger than a single-phase porosity-based REV.

This links to the observed cluster size distribution both in this work (Figs. 7 and 8) and in the literature [24,36,37,39,40] with regard to the apparent power-law distribution and the non-power-law tail for large clusters. According to a powerlaw distribution of cluster number $N(s) \propto s^{-\tau}$, it should follow that the cluster volume must scale as $s N(s) \propto s^{1-\tau}$. This means that the volume fraction of the largest clusters $(s \rightarrow \infty)$ should vanish $\left(s^{1-\tau} \rightarrow 0\right)$ for percolation exponents $\tau$. In other words, even though percolation does not provide an upper bound for large clusters, a two-phase REV may still be defined due to the ever-insignificant contribution of large clusters to saturation, should a power law hold throughout [46]. Yet observed 


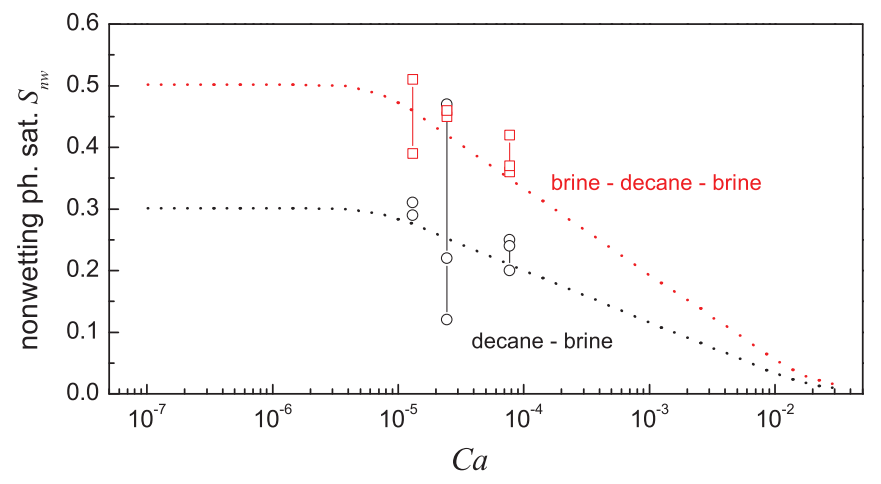

FIG. 12. (Color online) Influence of interfacial tension on the total nonwetting phase saturation after imbibition of 1, 5, and $30 \mathrm{PV}$. The data are plotted as a function of the capillary number varied by the interfacial tension between both fluid phases. The red and black symbols represent different flooding sequences: black circles: $n$-decane (presaturation) to brine; red squares: brine (presaturation) to $n$-decane to brine. The capillary desaturation curve (lower-black) [5] and the same curve scaled by a factor of $5 / 3$ (upper-red) are plotted for comparison.

cluster size distributions with non-power-law tails are found in porous media flow, which further appear to depend on the morphology of the pore space; media with low pore-throat to pore-body aspect ratios seem to have more clear non-powerlaw tails than sandstone rock [46], as seen in the present work. This signifies that large clusters become significant and do in fact affect the two-phase REV.

\section{E. Influence of surfactant}

For some experiments we added surfactant (DTAB) to the brine phase in order to reduce the interfacial tension and as a consequence to increase the capillary number $\mathrm{Ca}$. $\mathrm{Ca}$ was varied from $10^{-5}$ to $10^{-4}$ over the range of capillary de-saturation. The capillary desaturation curve [5] as well as the experimental nonwetting phase saturations after 1, 5, and 30 PV of brine imbibition are plotted in Fig. 12. The data are from two types of experiment, the first starting at initial brine saturation followed by a primary drainage of $200 \mathrm{PV} n$-decane and a subsequent imbibition process, and the second starting at a $100 \% n$-decane saturated core (no initial brine) followed by brine imbibition.

The nonwetting phase saturations for the two different initial states show a similar behavior and follow approximately the trend of the capillary desaturation curve. The absolute saturation of the brine and $n$-decane presaturated experiments tended however to be larger, compatible with the desaturation curve scaled by a factor of 5/3. The absolute observed nonwetting phase saturation might be a property of the individual sample and the finite volume injected, i.e., true residual saturation is not yet reached.

The data of the individual experiments show a large scatter, which is only partly related to the additional displacement by the injection of more brine. The scatter could be mainly related to the fact that the field of view is not sufficient to measure macroscopic saturations (see REV discussion in Sec. III D). The total measured saturation depends on whether the large clusters have moved into or out of the field of view in the specific measurement.

\section{SUMMARY AND CONCLUSIONS}

We performed two-phase flow experiments in a porous medium to investigate the nonwetting phase distribution during drainage and imbibition on the pore scale. The distribution was imaged by in situ $\mu \mathrm{CT}$ scanning with a pore-scale resolution and a maximized field of view. Large nonwetting-phase clusters were observed, dispersed from the size of a single pore to the size of several-hundred pore volumes and pore lengths. The maximum cluster size was estimated to be much larger than the obtainable field of view, compatible with the experimental data. The cluster size distribution shows a signature of a power-law behavior between 10 and $100 \mu \mathrm{m}$ with an experimental cutoff thereafter. The consequence of the experimental cutoff is that the largest clusters are statistically not well represented, which is a principal problem. However, the largest clusters contain the majority of the total nonwetting phase, with the volume of the largest cluster observed varying from $65 \%$ up to $99 \%$ of the total nonwetting phase volume. This seems to be currently a limitation of $\mu \mathrm{CT}$ scanning, which is the preferred if not only method to image fluid distributions in 3D porous media; physically relevant clusters are only deficiently represented, while clusters that are statistically well represented are physically less relevant. The question of what constitutes a representative elementary volume of two-phase flow is not only relevant for the scale of experiment and imaging, but also for numerical modeling of pore-scale processes, since the respective volumes at a sufficient resolution require a yet or not always feasible computational power.

\section{ACKNOWLEDGMENTS}

R. Hilfer is acknowledged for the valuable discussion about the estimation of the maximum cluster length. R. Walshe is acknowledged for proofreading the manuscript. We thank J. Beljaars, P. Legerstee, the Experimental Services Team, and the Rock \& Fluid Physics Team for their excellent technical support. Martin Blunt is acknowledged for helpful discussions related to cluster-size distributions and their physical relevance. We thank Arjen Cense for his help with the $P c$ estimation using eCore.
[1] H. P. G. Darcy, Les fontaines publiques de la ville de Dijon: Exposition et application des principes à suivre et des formules à employer dans les questions de distribution d'eau: Ouvrage terminé par un appendice relatif aux fournitures d'eau de plusieurs villes, au filtrage des eaux et à la fabrication des tuyaux de fonte, de plomb, de tole et de bitume (Dalmont, Paris, 1856).

[2] S. M. Hassanizadeh and W. G. Gray, Adv. Water Res. 16, 53 (1993).

[3] F. Kalaydjian, Transp. Porous Media 2, 537 (1987). 
[4] J. Bear, Dynamics of Fluids in Porous Media (Dover, New York, 1988).

[5] L. Lake, Enhanced Oil Recovery (Prentice Hall, New York, 1989).

[6] J. Niessner, S. Berg, and S. M. Hassanizadeh, Transp. Porous Media 88, 133 (2011).

[7] D. B. Das and S. M. Hassanizadeh, eds., Perface on Upscaling Multiphase Flow in Porous Media: From Pore to Core and Beyond (Springer, New York, 2005).

[8] P. Spanne, J. F. Thovert, C. J. Jacquin, W. B. Lindquist, K. W. Jones, and P. M. Adler, Phys. Rev. Lett. 73, 2001 (1994).

[9] J. Coenen, E. Tchouparova, and X. Jing, Soc. Core Anal. Conf. Paper SCA2004-36 (2004).

[10] A. O. Olafuyi, A. P. Sheppard, C. H. Arns, R. M. Sok, Y. Cinar, and M. A. Knackstedt, Soc. Petrol. Eng. 99897 (2006).

[11] S. Berg, H. Ott, S. Klapp, A. Schwing, R. Neitler, N. Brussee, A. Makurat, L. Leu, F. Enzmann, J.-O. Schwartz et al., Proc. Natl. Acad. Sci. USA 110, 3755 (2013).

[12] M. Feser, J. Gelb, H. Chang, H. Cui, F. Duewer, S. H. Lau, A. Tkachuk, and W. Yun, Meas. Sci. Technol. 19, 094001 (2008).

[13] C. Sisk, E. Diaz, J. Walls, A. Grader, and M. Suhrer, Soc. Petrol. Eng. 134582, 1 (2010).

[14] M. Appel (personal communication) (2013).

[15] M. J. Blunt, M. D. Jackson, M. Piri, and P. H. Valvatne, Adv. Water Resour. 25, 1069 (2002).

[16] H. Okabe and M. J. Blunt, Phys. Rev. E 70, 066135 (2004).

[17] P. Mostaghimi, B. Bijeljic, and M. J. Blunt, Soc. Petrol. Eng. 135261 (2010).

[18] M. E. Coles, R. D. Hazlett, P. Spanne, W. E. Soll, E. L. Muegge, and K. W. Jones, J. Petrol. Sci. Eng. 19, 55 (1998).

[19] M. E. Coles, R. D. Hazlett, E. L. Muegge, K. W. S. Jones, B. Andrews, B. Dowd, P. Siddons, A. Peskin, P. Spanne, and S. W. E., SPE Reservoir Eval. Eng. August, 288 (1998).

[20] S. Youssef, D. Bauer, S. Bekri, E. Rosenberg, and O. Vizika, Soc. Core Anal. Conf. Paper SCA 2009-17 (2009).

[21] S. Youssef, D. Bauer, S. Bekri, E. Rosenberg, and O. Vizika, Soc. Petrol. Eng. 135194 (2010).

[22] M. Kumar, T. J. Senden, A. P. Sheppard, J. P. Middleton, and M. A. Knackstedt, Soc. Core Anal. Conf. Paper SCA2009-16 (2009).
[23] M. Kumar, J. P. Middleton, A. P. Sheppard, T. J. Senden, and M. A. Knackstedt, in Proceedings of the International Petroleum Technology Conference (Doha, 2009), IPTC 14001.

[24] S. Iglauer, S. Favretto, G. Spinelli, G. Schena, and M. J. Blunt, Phys. Rev. E 82, 056315 (2010).

[25] D. G. Avraam and A. C. Payatakes, J. Fluid Mech. 293, 207 (1995).

[26] K. Nordahl and P. S. Ringrose, Math. Geosci. 40, 753 (2008).

[27] M. Porter, D. Wildenschild, G. Grant, and J. Gerhard, Water Resour. Res. 46, W08512 (2010).

[28] D. Wildenschild, R. T. Armstrong, A. L. Herring, I. M. Young, and J. W. Carey, Energy Procedia 4, 4945 (2011).

[29] T. R. Armstrong, M. L. Porter, and D. Wildenschild, Adv. Water Resour. 46, 55 (2012).

[30] T. R. Armstrong (unpublished).

[31] A. Georgiadis, G. C. Maitland, J. P. M. Trusler, and A. Bismarck, J. Chem. Eng. Data 55, 4168 (2010).

[32] A. Georgiadis, F. Llovell, A. Bismarck, F. J. Blas, A. Galindo, G. C. Maitland, J. P. M. Trusler, and G. Jackson, J. Supercrit. Fluids 55, 743 (2010).

[33] A. Georgiadis, G. C. Maitland, J. P. M. Trusler, and A. Bismarck, J. Chem. Eng. Data 56, 4900 (2011).

[34] X. Li, E. Boek, G. C. Maitland, and J. P. M. Trusler, J. Chem. Eng. Data 57, 1078 (2012).

[35] X. Li, E. S. Boek, G. C. Maitland, and J. P. M. Trusler, J. Chem. Eng. Data 57, 1369 (2012).

[36] D. Wilkinson, Phys. Rev. A 34, 1380 (1986).

[37] M. Blunt, M. J. King, and H. Scher, Phys. Rev. A 46, 7680 (1992).

[38] C. D. Lorenz and R. M. Ziff, Phys. Rev. E 57, 230 (1998).

[39] S. Iglauer, M. Ferno, P. Shearing, and M. J. Blunt, J. Colloid Interface Sci. 375, 187 (2012).

[40] C. Pentland, S. Iglauer, O. Ghabri, K. Okada, and T. Suekane, Soc. Petrol. Eng. 158516 (2012).

[41] C. S. A. Clauset and M. Newman, SIAM Rev. 51, 661 (2009).

[42] Y. Virkar and A. Clauset arXiv:1208.3524 (2012).

[43] M. Prodanovic, W. Lindquist, and R. Seright, J. Colloid Interface Sci. 298, 282 (2006).

[44] M. Prodanovic, W. P. Lindquist, and R. S. Seright, Adv. Water Res. 30, 214 (2007).

[45] R. Hilfer and P. E. Øren, Transp. Porous Media 22, 53 (1996).

[46] M. J. Blunt (personal communication) (2013). 